\title{
InterCriteria Analysis applied to human resources in science and technology
}

\author{
George Rusev and Veselina Bureva \\ "Prof. Dr. Assen Zlatarov" University \\ "Prof. Yakimov" Blvd., Burgas 8010, Bulgaria \\ e-mails: grussev71@gmail.com, vbureva@btu.bg
}

Received: 20 March 2019

Revised: 25 April 2019

Accepted: 1 May 2019

\begin{abstract}
In the current paper an application of the Inter Criteria approach to data on education inflows obtained from Eurostat's Education database (HRST-Human Resources in Science \& Technology) is presented. The aim is to analyze the correlations between 35 countries (the EU Member States and available Candidate Countries and EFTA countries) against the years 2008-2017.
\end{abstract}

Keywords: InterCriteria Analysis, Eurostat's education database, Human Resources in Science \& Technology.

2010 Mathematics Subject Classification: 03 E72.

\section{Introduction}

In the presented paper, we apply in sequential steps the new approach of inter-criteria analysis (ICA) on Eurostat data-HRST, using data from International Standard Classification of Education (ISCED), developed by UNESCO [15]. Due to increasing demand for internationally comparable indicators on education and a mounting complexity in the educational programmes on offer in different countries, the original standard, developed in 1976, has revised and updated in 1997. In 2011 a new revision of ISCED classification had taken place but the transition was not yet achieved in the Education section of Eurostat. Consequently, in the tables presenting the inflows from education, the ISCED1997 is still employed since 1997 onwards. HRST domain focuses on the tertiary level of education, which in the ISCED1997 classification corresponds to levels 5A, 5B and 6. HRST domain focuses on tertiary level of education, which in the ISCED1997 classification corresponds to levels 5A, 5B 
and 6. The description of the content of the levels forming tertiary education is given below. The International Standard Classification of Education (ISCED1997)

- ISCED level 5A: programmes that are largely theoretically based and are intended to provide sufficient qualifications for gaining entry into advanced research programmes and professions with high skill requirements;

- ISCED level 5B: programmes that are generally more practical/technical/occupationally specific than ISCED 5A programmes;

- ISCED level 6: this level is reserved for tertiary programmes that lead to the award of an advanced research qualification. The programmes are devoted to advanced study and original research.

Canberra Manual describes the HRST as persons who have completed tertiary education in S\&T fields of study. The operational definition of HRST doesn't restrict the population to those who graduated in S\&T field. All persons who achieved the tertiary level of education are considered HRST. However, some statistics on participation and graduation from tertiary education are available according to field of study. Useful methods for processing the statistical data are presented in $[17,18]$ with examples in the area of the petrol refining industry and management of primary energy sources. A selection of tables presents either HRST stocks or flows by focusing on fields with scientific and engineering programmes [15].

\section{Application of intercriteria analysis over human resources in science and technology}

In the presented paper we analyze the input dataset from HRST statistics. The digits are related to the 'percentage of employed population'. The data covers annual real (graduation) and potential (participation) inflows from the education system into HRST at the national level, by gender, some domains of study relevant to science and technology and also according to the citizenship of the students. Those people graduating at the ISCED 6 level will, however, already be a part of the HRST stocks since ISCED 6 refers to advanced research for which it is necessary to have a first degree [15].

The ICA for decision making, based on index matrices IMs and intuitionistic fuzzy sets IFSs is introduced by Atanassov et al. in [5, 6]. Intuitionistic fuzzy sets were first defined by Atanassov [3, 7] as an extension of the concept of fuzzy sets defined by L. Zadeh [23]. The theory of index matrices was introduced in [4].The InterCriteria Analysis (ICA) is designed for datasets comprising evaluations, or measurements of multiple objects against multiple criteria. In the initial formulation of the method, the aim was to detect correlations between the criteria, in order to eliminate future evaluations/measurements against some of the criteria, which exhibit high enough correlations with others. The theoretical investigations for ICA are published in $[2,11,13,21]$. The series of papers present the applications of the ICA in the different fields of science: universities rankings [12,14], neural networks preprocessing procedure [19], genetic algorithms [1], economic investigations [8-10], medical investigations [22], chemistry applications [20] and etc. In our work for applying ICA to HRST data, we have been interested to detect the eventual correlations between the education inflow data in 35 
countries. The input data for the application of the InterCriteria Analysis over HRST-Human Resources in Science \& Technology is presented in Table 1.

\begin{tabular}{|c|c|c|c|c|c|c|c|c|c|c|}
\hline Country/Year & 2008 & 2009 & 2010 & 2011 & 2012 & 2013 & 2014 & 2015 & 2016 & 2017 \\
\hline 1 Belgium & 13.1 & 11.7 & 12 & 12.2 & 11 & 10.7 & 10.5 & 10.4 & 9.9 & 9.9 \\
\hline 2 Bulgaria & 12.7 & 11.7 & 11.1 & 11.3 & 11.6 & 11 & 11.1 & 11.2 & 10.7 & 10.4 \\
\hline 3 Czechia & 17.4 & 16.4 & 15.9 & 16.9 & 17.8 & 16.9 & 17.5 & 17.7 & 17.9 & 18.2 \\
\hline 4 Denmark & 11.2 & 10.3 & 10.3 & 10.7 & 10.2 & 10 & 9.9 & 10.2 & 10.3 & 10.2 \\
\hline 5 Germany & 15.4 & 15.5 & 15 & 15 & 14.9 & 14.7 & 15.1 & 15.1 & 14.7 & 14.8 \\
\hline 6 Estonia & 13.3 & 11.7 & 12 & 12.7 & 12.2 & 11.5 & 11.5 & 12.3 & 12.3 & 12.8 \\
\hline 7 Ireland & 10.8 & 10.6 & 10.5 & 10.2 & 10 & 10 & 10.2 & 10.4 & 11.1 & 10.8 \\
\hline 8 Greece & 7.2 & 6.3 & 5.9 & 6 & 5.7 & 5.4 & 5.1 & 5.8 & 6.5 & 6.1 \\
\hline 9 Spain & 12.6 & 11.6 & 11.2 & 11.4 & 11.5 & 11.3 & 11.2 & 11.2 & 11 & 11.3 \\
\hline 10 France & 12.9 & 12 & 11.7 & 12.3 & 12.2 & 11.8 & 11.3 & 11.3 & 11.5 & 11.2 \\
\hline 11 Croatia & 12.6 & 11.5 & 11.5 & 11.9 & 12.2 & 12.7 & 10.9 & 11.3 & 10.2 & 11.2 \\
\hline 12 Italy & 13.1 & 12.8 & 12.2 & 13.5 & 13.6 & 13.7 & 14.2 & 14.3 & 14.4 & 14.2 \\
\hline 13 Cyprus & 4.2 & 4.3 & 4.4 & 4.1 & 4.1 & 3.8 & 4.1 & 3.8 & 4.2 & 4.4 \\
\hline 14 Latvia & 9.7 & 7.8 & 6.9 & 7.1 & 8.1 & 8.4 & 7.8 & 8.1 & 8.7 & 7.5 \\
\hline 15 Lithuania & 10.5 & 9.2 & 8.9 & 9.6 & 9.6 & 9.3 & 9.8 & 10.3 & 9.5 & 9.2 \\
\hline 16 Luxembourg & 3.3 & 3.5 & 3.1 & 3.4 & 3.8 & 3.1 & 3.5 & 3.6 & 3 & 2 \\
\hline 17 Hungary & 12.1 & 11.6 & 10.9 & 11 & 11.2 & 11.6 & 11.7 & 12.3 & 12.3 & 13.7 \\
\hline 18 Malta & 9.8 & 9.2 & 10 & 9.4 & 8.4 & 8.4 & 8.3 & 7.8 & 8.4 & 8.8 \\
\hline 19 Netherlands & 6.8 & 6.4 & 6.3 & 7.4 & 7.2 & 6.9 & 6.9 & 7 & 7.1 & 6.8 \\
\hline 20 Austria & 12.7 & 12.2 & 12.6 & 13.6 & 13.6 & 13.9 & 14 & 14.5 & 14.2 & 13.6 \\
\hline 21 Poland & 12.3 & 11.3 & 11.1 & 11.9 & 11.9 & 12.1 & 12.3 & 12.9 & 13.7 & 14.3 \\
\hline 22 Portugal & 7.4 & 7.5 & 6.8 & 9.7 & 9.6 & 9.2 & 9.6 & 10 & 10.3 & 10 \\
\hline 23 Romania & 15.3 & 13.5 & 12.7 & 12.8 & 13.1 & 13 & 12.2 & 11.7 & 11.7 & 11.9 \\
\hline 24 Slovenia & 17.7 & 17.6 & 16.9 & 15.6 & 14.6 & 14 & 14.9 & 15.8 & 17.4 & 16.7 \\
\hline 25 Slovakia & 15.8 & 14.8 & 13.6 & 14.9 & 14.1 & 13.3 & 13.6 & 15 & 14.5 & 14.4 \\
\hline 26 Finland & 13.1 & 12.9 & 12.5 & 12 & 12.7 & 12.7 & 11.8 & 11.6 & 11.7 & 11.3 \\
\hline 27 Sweden & 9 & 8.5 & 7.9 & 7.9 & 7.9 & 7.9 & 7.9 & 8.2 & 8.2 & 8 \\
\hline 28 United Kingdom & 9.4 & 7.5 & 7.8 & 8.1 & 8.3 & 8 & 8.1 & 7.9 & 8 & 7.8 \\
\hline 29 Iceland & 4.5 & 4.7 & 4.4 & 5.3 & 6.3 & 6.3 & 5.7 & 4.9 & 5.9 & 5.1 \\
\hline 30 Norway & 6.5 & 6.3 & 6 & 6.6 & 6.2 & 6.1 & 6.4 & 6.5 & 6.4 & 6.2 \\
\hline 31 Switzerland & 11.3 & 11.1 & 11.1 & 11.5 & 11.2 & 11.2 & 10.8 & 10.6 & 10.7 & 10.8 \\
\hline 32 Montenegro & $:$ & . & . & 5.6 & 4.3 & 4.6 & 4.4 & 3.6 & 3.5 & 3.9 \\
\hline 33 North Macedonia & $:$ & $:$ & : & 11 & 10.6 & 10.8 & 10.5 & 11.2 & 10.9 & 11.5 \\
\hline 34 Serbia & $:$ & $:$ & 12.5 & 10.6 & 11.1 & 12.7 & 11.3 & 11 & 11.5 & 12.3 \\
\hline 35 Turkey & $:$ & 12.6 & 13.3 & 12.3 & 11.9 & 12.2 & 12.5 & 12.4 & 11.4 & 11.5 \\
\hline
\end{tabular}

Table 1. People with tertiary education (ISCED) and/or employed in science and technology from 15 to 74 years-percentage of total employment. 


\subsection{Application of InterCriteria Analysis over human resources in science and technology for determining the correlations between the countries}

The ICA is applied over the dataset of HRST-Human Resources in Science \& Technology to determine possible dependencies or opposites between the countries for 10 years. The dataset has several missing values for the counties Turkey, Serbia, North Macedonia and Montenegro. The missing data is for the years 2008-2010. This information will be signed with the digit 0 . The ICA is applied using ICrAData software which is developed by Ikonomov et al. [16].

The matrices containing the degrees of membership ( $\mu$-values) and degrees of nonmembership ( $v$-values) for the current investigation are presented in Table 2 and Table 3, respectively. The matrices contain the values for the degrees of correspondence and the degrees of non-correspondence present the dependencies between the selected 35 countries according to the years (2008-2017). The highest numbers present higher dependencies between the countries, otherwise the selected objects are not dependent or have the opposite behaviour.

\begin{tabular}{|c|c|c|c|c|c|c|c|c|c|c|c|c|c|c|c|c|c|c|c|c|c|c|c|c|c|c|c|c|c|c|c|}
\hline 0.8 & & & & & & & & 4 & & & 0.5 & & & & & & 0 & 0.20 & 0 & & 30 & 0.50 . & 0.6 & & & 0.30 & 50 & 0.8 & $\begin{array}{ll}4 & 0.2\end{array}$ & & \\
\hline & 0.3 & & & & 0 & & & & & & & & & & & & 0.50 & 0.30 & 0 & & 0.80 & 0.60. & 0.7 & 5 & & ( & & & & & \\
\hline .3 & & & & & & & & & & & & & & & & & & & & & & & & & & & & & & & \\
\hline 0.6 & 0 & & & & & & & & & & & & & & & & & & & & & & 0.7 & & & & & & & & \\
\hline & 0.3 & & & & & & & & & 3 & 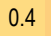 & & & 0.6 & 4 & & & 0.30 & 3 & & & 0. & 0.6 & & & 0.2 & & & & & 0 \\
\hline & & & & & & 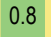 & & 0.5 & & 5 & 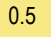 & & & 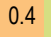 & 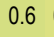 & 0.6 & 0 & 0.4 & 0.6 & & 0.50 & 0.60 . & $0.8 \quad 0.4$ & 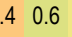 & 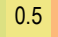 & 30 & 0.6 & & & 0.3 & 0 \\
\hline & & & & & & & & & 0 & 5 & j & & & & & & & 0.40 & 0.6 & & & 0 & & & & & & & & & \\
\hline & & & & 0.8 & 0 & & & & & 0.4 & 0.6 & & & & & & & 0.3 & & & & 0. & & & & & & & & & \\
\hline & & & & & & & & & & 0.2 & & & & & & & & $0 ? 2$ & & & & 0.50 & & & & & & & & & \\
\hline & & & & & & & & & & & & & & & & & & 0.30 & & & & 0.60 . & & & & & & & & & \\
\hline & & & & & & 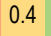 & & & & 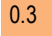 & 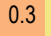 & & & 5 & & & & 0.30 & 0.30 & & 0 & 0.4 & $\begin{array}{ll}0.5 & 0\end{array}$ & & j & 0.40 & & & & & \\
\hline 0. & & & & 0.5 & & $t$ & & ( & & & 0.2 & & & 0.4 & & & & 0.80 & 80 & 0.8 & & 0.40 . & 0.5 & & & & & & & & 0 \\
\hline & & & & & & & & & & 0.2 & & & & & & & & 0.2 & & & & 0.60 . & 0. & & & & & & & & \\
\hline & & & & & & & & & & & & & & & & & & & & & & & & & & & & & & & \\
\hline & & & & & & & & & & 0.6 & & & & & & & & 0.60 & 0.60 & & & 0 & & & & & & & & & \\
\hline & & & & & & & & & & $t$ & ? & & & & 0.4 & & & 0.5 & 30 & & & 0.4 & 0.5 & & 3 & 50 & & & & & 0 \\
\hline & & & & & & & & & & 0.8 & & & & 0 & & & & 0.6 & 90 & & & $\begin{array}{ll}0.6 & 0 .\end{array}$ & 0. & & 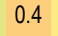 & 0.50 & & & & & \\
\hline & & & & & & & & & & & & & & & & & & . & & & & & & & & & & & & & \\
\hline & & & & & & & & & & & & & & & & & & & & & & & & & & & & & & & \\
\hline 0.3 & & & & & & & & & & 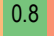 & . & & & & & & & & 0.70 & & & 0 & & & & & & & & & \\
\hline 0.3 & & & & & & & & ( & & 3 & & 0.6 & & 3 & 9 & ( & 0 & 0.7 & & 7 & 0 & 0.50 . & 0.5 & 5 & 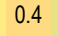 & 0.60 & 5 & & 50 & & 0 \\
\hline & & & & & & & & & & & & & & & & & 0 & 0.70 & & & 0 & 0.50 . & 0.6 & & & 0 & 0.6 & & & & \\
\hline & & & & & & & & & & & & & & & & & & & & & & & & & & & & & & & \\
\hline & & & & & & & & & & & & & & & & & & & & & & & & & & & & & & & \\
\hline 078 & & & & & & & & & & 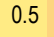 & & & & 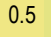 & 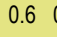 & 0.6 & 0 & 0.40 & 50 & 0.6 & 0 & 0. & 0.5 & 5 & & 0.30 & 80 & & & & \\
\hline 0.8 & & & & & & & & & & ? & 4 & & & c & & 7 & 0.40 & 0.20 & 0.20 & 0.2 & 0.90 & 0.5 & & & & 0.50 & 0.4 & & & & \\
\hline & & & & & & & & & & & & & & & & & & & & & & 0.70 . & 0.1 & & & & & & & & \\
\hline & & & & & & & & & & & & & & & & & & & & & & & & & & & & & & & \\
\hline & & & & & & & & & & & & & & & & & & & & & & $0 ?$ & 03 & & & & & & & & \\
\hline & & & & & & & & & & & & & 0.7 & c & & 0.4 & & 0.60 & & & 0.40 & 6 & $\begin{array}{lll}0.8 & 0.4\end{array}$ & .4 & 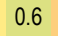 & & & & & & \\
\hline 0. & & & & & & & & & & & & & & & & & & & & & & 0.4 & & .8 & & .50 & & & & & \\
\hline & & & & 0.4 & & & & ( & & 0.0 & & & & 0.5 & 0.4 & 0.04 & 0.10 & 0.00 & 0.54 & 0.54 & 0.78 & 0.1 & 0.7 & .1 & 0.6 & 0.10 & 0.0 & & 0.1 & 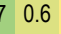 & \\
\hline & & & & & & & & & & & & & & & & & & & & & & & & & & & & & & & \\
\hline & & & & & & & & & & & & & & & & & & & & & & & & & & & & & & & \\
\hline & & & & & & & & & & & & & & & & & & & & & & & & & & & & & & & \\
\hline
\end{tabular}

Table 2. Results from the application ICA over the input data - degrees of membership $\mu$ 
$\begin{array}{llllllllllllllllllllllllllllllllllllll}0.2 & 0.8 & 0.2 & 0.3 & 0.4 & 0.5 & 0.4 & 0.2 & 0.1 & 0.2 & 0.8 & 0.4 & 0.5 & 0.4 & 0.4 & 0.8 & 0.2 & 0.5 & 0.7 & 0.8 & 0.8 & 0.2 & 0.4 & 0.4 & 0.2 & 0.4 & 0.3 & 0.6 & 0.4 & 0.2 & 0.5 & 0.7 & 0.7 & 0.4\end{array}$ $\begin{array}{lllllllllllllllllllllllllllllllllllllll}0.2 & 0.6 & 0.2 & 0.2 & 0.4 & 0.5 & 0.4 & 0.2 & 0.2 & 0.3 & 0.7 & 0.4 & 0.4 & 0.3 & 0.2 & 0.6 & 0.4 & 0.4 & 0.6 & 0.7 & 0.7 & 0.2 & 0.4 & 0.3 & 0.2 & 0.3 & 0.3 & 0.6 & 0.3 & 0.3 & 0.6 & 0.7 & 0.8 & 0.5\end{array}$ $\begin{array}{llllllllllllllllllllllllllllllllllllllll}0.8 & 0.6 & & 0.5 & 0.6 & 0.3 & 0.4 & 0.5 & 0.5 & 0.6 & 0.6 & 0.2 & 0.4 & 0.3 & 0.4 & 0.5 & 0.2 & 0.6 & 0.3 & 0.2 & 0.1 & 0.2 & 0.6 & 0.5 & 0.4 & 0.7 & 0.3 & 0.4 & 0.3 & 0.4 & 0.6 & 0.4 & 0.2 & 0.4 & 0.8\end{array}$ $\begin{array}{llllllllllllllllllllllllllllllllllll}0.2 & 0.2 & 0.5 & & 0.3 & 0.2 & 0.2 & 0.1 & 0.2 & 0.2 & 0.3 & 0.6 & 0.2 & 0.4 & 0.4 & 0.5 & 0.5 & 0.1 & 0.4 & 0.6 & 0.5 & 0.5 & 0.3 & 0.2 & 0.2 & 0.3 & 0.1 & 0.4 & 0.6 & 0.3 & 0.3 & 0.6 & 0.5 & 0.6 & 0.5\end{array}$ $\begin{array}{lllllllllllllllllllllllllllllllllllllllll}0.3 & 0.2 & 0.6 & 0.3 & 0.4 & 0.4 & 0.4 & 0.3 & 0.4 & 0.4 & 0.6 & 0.4 & 0.5 & 0.3 & 0.3 & 0.5 & 0.4 & 0.6 & 0.6 & 0.6 & 0.6 & 0.3 & 0.3 & 0.3 & 0.4 & 0.2 & 0.4 & 0.7 & 0.3 & 0.4 & 0.6 & 0.6 & 0.8 & 0.3\end{array}$ $\begin{array}{lllllllllllllllllllllllllllllllllllllll}0.4 & 0.4 & 0.3 & 0.2 & 0.4 & 0.3 & 0.2 & 0.4 & 0.4 & 0.5 & 0.5 & 0.3 & 0.4 & 0.4 & 0.6 & 0.4 & 0.3 & 0.4 & 0.5 & 0.3 & 0.4 & 0.5 & 0.3 & 0.2 & 0.6 & 0.2 & 0.4 & 0.6 & 0.3 & 0.4 & 0.5 & 0.3 & 0.6 & 0.7\end{array}$ $\begin{array}{lllllllllllllllllllllllllllllllllllllll}0.5 & 0.5 & 0.4 & 0.2 & 0.4 & 0.3 & & 0.1 & 0.5 & 0.5 & 0.6 & 0.4 & 0.2 & 0.4 & 0.4 & 0.6 & 0.2 & 0.4 & 0.6 & 0.5 & 0.3 & 0.4 & 0.6 & 0.1 & 0.3 & 0.5 & 0.1 & 0.6 & 0.7 & 0.4 & 0.6 & 0.7 & 0.4 & 0.5 & 0.5\end{array}$ $\begin{array}{llllllllllllllllllllllllllllllllllllll}0.4 & 0.4 & 0.5 & 0.1 & 0.4 & 0.2 & 0.1 & 0.3 & 0.4 & 0.5 & 0.6 & 0.3 & 0.4 & 0.5 & 0.6 & 0.4 & 0.3 & 0.5 & 0.6 & 0.4 & 0.4 & 0.4 & 0.1 & 0.2 & 0.4 & 0.1 & 0.5 & 0.7 & 0.3 & 0.4 & 0.7 & 0.5 & 0.7 & 0.7\end{array}$ $\begin{array}{llllllllllllllllllllllllllllllllllllllll}0.2 & 0.2 & 0.5 & 0.2 & 0.3 & 0.4 & 0.5 & 0.3 & & 0.2 & 0.2 & 0.7 & 0.4 & 0.4 & 0.4 & 0.3 & 0.5 & 0.2 & 0.5 & 0.7 & 0.6 & 0.6 & 0.1 & 0.4 & 0.3 & 0.2 & 0.3 & 0.3 & 0.5 & 0.4 & 0.2 & 0.4 & 0.6 & 0.7 & 0.5\end{array}$ $\begin{array}{llllllllllllllllllllllllllllllllllllll}0.1 & 0.2 & 0.6 & 0.2 & 0.4 & 0.4 & 0.5 & 0.4 & 0.2 & & 0.2 & 0.7 & 0.4 & 0.4 & 0.4 & 0.4 & 0.7 & 0.2 & 0.4 & 0.6 & 0.6 & 0.6 & 0.2 & 0.4 & 0.4 & 0.1 & 0.3 & 0.3 & 0.5 & 0.4 & 0.1 & 0.5 & 0.6 & 0.6 & 0.6\end{array}$ $\begin{array}{llllllllllllllllllllllllllllllllllll}0.2 & 0.3 & 0.6 & 0.3 & 0.4 & 0.5 & 0.6 & 0.5 & 0.2 & 0.2 & & 0.6 & 0.5 & 0.4 & 0.4 & 0.4 & 0.6 & 0.3 & 0.5 & 0.6 & 0.6 & 0.7 & 0.2 & 0.6 & 0.5 & 0.2 & 0.4 & 0.3 & 0.5 & 0.5 & 0.2 & 0.4 & 0.6 & 0.5 & 0.5\end{array}$ \begin{tabular}{llllllll|lll|lllllllllllllllllllllllll}
0.8 & 0.7 & 0.2 & 0.6 & 0.6 & 0.5 & 0.4 & 0.6 & 0.7 & 0.7 & 0.6 & 0.6 & 0.3 & 0.4 & 0.5 & 0.2 & 0.8 & 0.3 & 0.1 & 0.1 & 0.1 & 0.8 & 0.6 & 0.5 & 0.7 & 0.3 & 0.5 & 0.3 & 0.4 & 0.7 & 0.4 & 0.2 & 0.4 & 0.6
\end{tabular} $\begin{array}{lllllllllllllllllllllllllllllllllllll}0.4 & 0.4 & 0.4 & 0.2 & 0.4 & 0.3 & 0.2 & 0.3 & 0.4 & 0.4 & 0.5 & 0.6 & 0.6 & 0.6 & 0.6 & 0.4 & 0.2 & 0.6 & 0.6 & 0.5 & 0.5 & 0.4 & 0.2 & 0.4 & 0.4 & 0.3 & 0.5 & 0.6 & 0.5 & 0.4 & 0.6 & 0.5 & 0.4 & 0.4\end{array}$ $\begin{array}{lllllllllllllllllllllllllllllllllllll}0.5 & 0.4 & 0.3 & 0.4 & 0.5 & 0.4 & 0.4 & 0.4 & 0.4 & 0.4 & 0.4 & 0.3 & 0.6 & 0.2 & 0.4 & 0.3 & 0.6 & 0.4 & 0.3 & 0.3 & 0.4 & 0.4 & 0.5 & 0.4 & 0.4 & 0.2 & 0.3 & 0.4 & 0.4 & 0.4 & 0.5 & 0.4 & 0.5 & 0.8\end{array}$ $\begin{array}{lllllllllllllllllllllllllllllllllllll}0.4 & 0.3 & 0.4 & 0.4 & 0.3 & 0.4 & 0.4 & 0.5 & 0.4 & 0.4 & 0.4 & 0.4 & 0.6 & 0.2 & & 0.3 & 0.4 & 0.6 & 0.3 & 0.3 & 0.3 & 0.4 & 0.5 & 0.5 & 0.3 & 0.5 & 0.3 & 0.2 & 0.4 & 0.2 & 0.4 & 0.4 & 0.4 & 0.7 & 0.6\end{array}$ $\begin{array}{llllllllllllllllllllllllllllllllllll}0.4 & 0.2 & 0.5 & 0.5 & 0.3 & 0.6 & 0.6 & 0.6 & 0.3 & 0.4 & 0.4 & 0.5 & 0.6 & 0.4 & 0.3 & 0.6 & 0.6 & 0.3 & 0.4 & 0.6 & 0.5 & 0.4 & 0.6 & 0.4 & 0.4 & 0.4 & 0.3 & 0.4 & 0.4 & 0.4 & 0.4 & 0.5 & 0.6 & 0.4\end{array}$ $\begin{array}{llllllllllllllllllllllllllllllllllllll}0.8 & 0.6 & 0.2 & 0.5 & 0.5 & 0.4 & 0.2 & 0.4 & 0.5 & 0.7 & 0.6 & 0.2 & 0.4 & 0.3 & 0.4 & 0.6 & 0.6 & 0.5 & 0.2 & 0.0 & 0.3 & 0.6 & 0.4 & 0.4 & 0.6 & 0.2 & 0.5 & 0.4 & 0.3 & 0.7 & 0.5 & 0.3 & 0.4 & 0.7\end{array}$ $\begin{array}{lllllllllllllllllllllllllllllllllllllll}0.2 & 0.4 & 0.6 & 0.1 & 0.4 & 0.3 & 0.4 & 0.3 & 0.2 & 0.2 & 0.3 & 0.8 & 0.2 & 0.6 & 0.6 & 0.6 & 0.6 & 0.6 & 0.8 & 0.6 & 0.7 & 0.3 & 0.3 & 0.4 & 0.3 & 0.3 & 0.5 & 0.7 & 0.5 & 0.2 & 0.6 & 0.6 & 0.5 & 0.5\end{array}$ $\begin{array}{llllllllllllllllllllllllllllllllllllll}0.5 & 0.4 & 0.3 & 0.4 & 0.6 & 0.4 & 0.6 & 0.5 & 0.5 & 0.4 & 0.5 & 0.3 & 0.6 & 0.4 & 0.3 & 0.3 & 0.5 & 0.6 & 0.2 & 0.4 & 0.2 & 0.6 & 0.6 & 0.4 & 0.5 & 0.5 & 0.2 & 0.2 & 0.3 & 0.4 & 0.2 & 0.2 & 0.5 & 0.6\end{array}$ $\begin{array}{lllllllllllllllllllllllllllllllllllllll}0.7 & 0.6 & 0.2 & 0.6 & 0.6 & 0.5 & 0.5 & 0.6 & 0.7 & 0.6 & 0.6 & 0.1 & 0.6 & 0.3 & 0.3 & 0.4 & 0.2 & 0.8 & 0.2 & 0.2 & 0.2 & 0.7 & 0.6 & 0.5 & 0.7 & 0.4 & 0.4 & 0.3 & 0.3 & 0.6 & 0.3 & 0.2 & 0.4 & 0.5\end{array}$ $\begin{array}{llllllllllllllllllllllllllllllllllllll}0.8 & 0.7 & 0.1 & 0.5 & 0.6 & 0.3 & 0.3 & 0.4 & 0.6 & 0.6 & 0.6 & 0.1 & 0.5 & 0.3 & 0.3 & 0.6 & 0.0 & 0.6 & 0.4 & 0.2 & & 0.2 & 0.7 & 0.4 & 0.4 & 0.7 & 0.3 & 0.4 & 0.4 & 0.4 & 0.6 & 0.4 & 0.2 & 0.4 & 0.7\end{array}$ $\begin{array}{lllllllllllllllllllllllllllllllllllllll}0.8 & 0.7 & 0.2 & 0.5 & 0.6 & 0.4 & 0.4 & 0.4 & 0.6 & 0.6 & 0.7 & 0.1 & 0.5 & 0.4 & 0.4 & 0.5 & 0.3 & 0.7 & 0.2 & 0.2 & 0.2 & 0.8 & 0.5 & 0.4 & 0.8 & 0.3 & 0.5 & 0.3 & 0.3 & 0.6 & 0.4 & 0.1 & 0.4 & 0.6\end{array}$ $\begin{array}{llllllllllllllllllllllllllllllllllllll}0.2 & 0.2 & 0.6 & 0.3 & 0.3 & 0.5 & 0.6 & 0.4 & 0.1 & 0.2 & 0.2 & 0.8 & 0.4 & 0.4 & 0.5 & 0.4 & 0.6 & 0.3 & 0.6 & 0.7 & 0.7 & 0.8 & 0.5 & 0.5 & 0.1 & 0.4 & 0.3 & 0.5 & 0.5 & 0.1 & 0.5 & 0.7 & 0.6 & 0.5\end{array}$ $\begin{array}{llllllllllllllllllllllllllllllllllll}0.4 & 0.4 & 0.5 & 0.2 & 0.3 & 0.3 & 0.1 & 0.1 & 0.4 & 0.4 & 0.6 & 0.6 & 0.2 & 0.5 & 0.5 & 0.6 & 0.4 & 0.3 & 0.6 & 0.6 & 0.4 & 0.5 & 0.5 & 0.2 & 0.4 & 0.1 & 0.6 & 0.8 & 0.4 & 0.5 & 0.8 & 0.6 & 0.6 & 0.6\end{array}$ $\begin{array}{llllllllllllllllllllllllllllllllllll}0.4 & 0.3 & 0.4 & 0.2 & 0.3 & 0.2 & 0.3 & 0.2 & 0.3 & 0.4 & 0.5 & 0.5 & 0.4 & 0.4 & 0.3 & 0.4 & 0.4 & 0.4 & 0.4 & 0.5 & 0.4 & 0.4 & 0.5 & 0.2 & & 0.5 & 0.1 & 0.4 & 0.7 & 0.2 & 0.5 & 0.6 & 0.4 & 0.8 & 0.6\end{array}$ $\begin{array}{llllllllllllllllllllllllllllllllllllll}0.2 & 0.2 & 0.7 & 0.3 & 0.4 & 0.6 & 0.5 & 0.4 & 0.2 & 0.1 & 0.2 & 0.7 & 0.4 & 0.4 & 0.5 & 0.4 & 0.6 & 0.3 & 0.5 & 0.7 & 0.7 & 0.8 & 0.1 & 0.4 & 0.5 & 0.3 & 0.3 & 0.5 & 0.5 & 0.2 & 0.6 & 0.8 & 0.6 & 0.5\end{array}$ $\begin{array}{llllllllllllllllllllllllllllllllllllll}0.4 & 0.3 & 0.3 & 0.1 & 0.2 & 0.2 & 0.1 & 0.1 & 0.3 & 0.3 & 0.4 & 0.3 & 0.3 & 0.2 & 0.3 & 0.4 & 0.2 & 0.3 & 0.5 & 0.4 & 0.3 & 0.3 & 0.4 & 0.1 & 0.1 & 0.3 & 0.4 & 0.6 & 0.2 & 0.4 & 0.6 & 0.4 & 0.6 & 0.5\end{array}$ $\begin{array}{lllllllllllllllllllllllllllllllllllllll}0.3 & 0.3 & 0.4 & 0.4 & 0.4 & 0.4 & 0.6 & 0.5 & 0.3 & 0.3 & 0.3 & 0.5 & 0.5 & 0.3 & 0.2 & 0.3 & 0.5 & 0.5 & 0.2 & 0.4 & 0.4 & 0.5 & 0.3 & 0.6 & 0.4 & 0.3 & 0.4 & 0.3 & 0.3 & 0.3 & 0.3 & 0.5 & 0.6 & 0.7\end{array}$ $\begin{array}{llllllllllllllllllllllllllllllllllllll}0.6 & 0.6 & 0.3 & 0.6 & 0.7 & 0.6 & 0.7 & 0.7 & 0.5 & 0.5 & 0.5 & 0.3 & 0.6 & 0.4 & 0.4 & 0.4 & 0.4 & 0.7 & 0.2 & 0.3 & 0.4 & 0.3 & 0.5 & 0.8 & 0.7 & 0.5 & 0.6 & 0.3 & 0.5 & 0.5 & 0.2 & 0.4 & 0.3 & 0.6\end{array}$ $\begin{array}{lllllllllllllllllllllllllllllllllllll}0.4 & 0.3 & 0.4 & 0.3 & 0.3 & 0.3 & 0.4 & 0.3 & 0.4 & 0.4 & 0.5 & 0.4 & 0.5 & 0.4 & 0.2 & 0.4 & 0.3 & 0.5 & 0.3 & 0.3 & 0.4 & 0.3 & 0.5 & 0.4 & 0.2 & 0.5 & 0.2 & 0.3 & 0.5 & 0.4 & 0.4 & 0.4 & 0.7 & 0.6\end{array}$ $\begin{array}{llllllllllllllllllllllllllllllllllllll}0.2 & 0.3 & 0.6 & 0.3 & 0.4 & 0.4 & 0.6 & 0.4 & 0.2 & 0.1 & 0.2 & 0.7 & 0.4 & 0.4 & 0.4 & 0.4 & 0.7 & 0.2 & 0.4 & 0.6 & 0.6 & 0.6 & 0.1 & 0.5 & 0.5 & 0.2 & 0.4 & 0.3 & 0.5 & 0.4 & 0.4 & 0.6 & 0.5 & 0.5\end{array}$ $\begin{array}{llllllllllllllllllllllllllllllllllll}0.5 & 0.6 & 0.4 & 0.6 & 0.6 & 0.5 & 0.7 & 0.7 & 0.4 & 0.5 & 0.4 & 0.4 & 0.6 & 0.5 & 0.4 & 0.4 & 0.5 & 0.6 & 0.2 & 0.3 & 0.4 & 0.4 & 0.5 & 0.8 & 0.6 & 0.6 & 0.6 & 0.3 & 0.2 & 0.4 & 0.4 & 0.3 & 0.4 & 0.4\end{array}$ $\begin{array}{llllllllllllllllllllllllllllllllllllllll}0.7 & 0.7 & 0.2 & 0.5 & 0.6 & 0.3 & 0.4 & 0.5 & 0.6 & 0.6 & 0.6 & 0.2 & 0.5 & 0.4 & 0.4 & 0.5 & 0.3 & 0.6 & 0.2 & 0.2 & 0.2 & 0.1 & 0.7 & 0.6 & 0.4 & 0.8 & 0.4 & 0.5 & 0.4 & 0.4 & 0.6 & 0.3 & 0.4 & 0.6\end{array}$ $\begin{array}{llllllllllllllllllllllllllllllllllllll}0.7 & 0.8 & 0.4 & 0.6 & 0.8 & 0.6 & 0.5 & 0.7 & 0.7 & 0.6 & 0.5 & 0.4 & 0.4 & 0.5 & 0.7 & 0.6 & 0.4 & 0.5 & 0.5 & 0.4 & 0.4 & 0.4 & 0.6 & 0.6 & 0.8 & 0.6 & 0.6 & 0.6 & 0.3 & 0.7 & 0.5 & 0.4 & 0.4 & 0.5\end{array}$ $\begin{array}{lllllllllllllllllllllllllllllllllllll}0.4 & 0.5 & 0.8 & 0.5 & 0.3 & 0.7 & 0.5 & 0.7 & 0.5 & 0.6 & 0.5 & 0.6 & 0.4 & 0.8 & 0.6 & 0.4 & 0.7 & 0.5 & 0.6 & 0.5 & 0.7 & 0.6 & 0.5 & 0.6 & 0.6 & 0.5 & 0.5 & 0.7 & 0.6 & 0.6 & 0.5 & 0.4 & 0.6 & 0.5\end{array}$

Table 3. Results from the application ICA over the input data - degree of non-membership $\nu$

The results of the ICA application over data for or Human Resources in Science \& Technology are visualized onto the intuitionistic fuzzy interpretational triangle (Fig.1). They are investigated and the following outcomes are obtained: 3 pairs of countries in positive consonance, 31 pairs of countries in weak positive consonance, 126 pairs of countries in weak dissonance, 218 pairs of countries in dissonance, 181 pairs of countries in strong dissonance, 54 pairs of countries in weak negative consonance and 7 pairs of countries in negative consonance. 


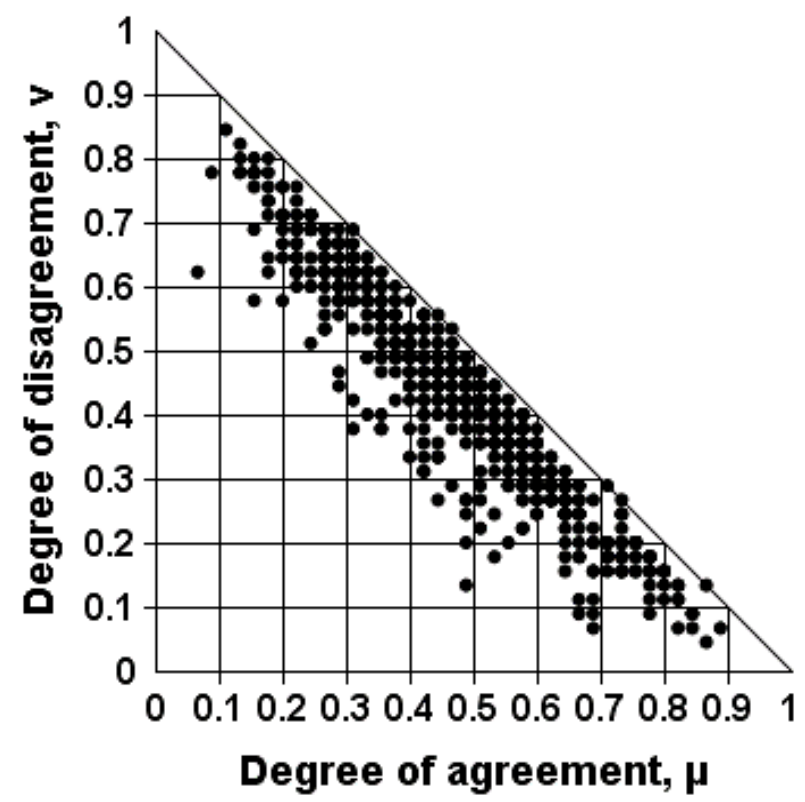

Figure 1. Results from application of ICA, plotted onto the intuitionistic fuzzy interpretational triangle

Obviously, the pairs of countries having the most similar trend of Human Resources in Science \& Technology are Ireland-Slovenia, Hungary-Poland and Romania-Finland. Therefore, the pairs of countries having opposite behavior according to the investigated area are: Belgium-Italy, Bulgaria-Serbia, Italy-Malta, Malta-Austria, Slovenia-Montenegro, Finland-North Macedonia and Sweden-Montenegro.

\subsection{Application of intercriteria analysis over human resources in science and technology for determining the correlations in the years}

Second application of the ICA over the data for Human Resources in Science \& Technology is applied to determine the trends and correlation between the years. The matrices containing the degrees of membership and the degrees of non-membership are presented on Tables 4 and 5, respectively.

\begin{tabular}{|l|c|c|c|c|c|c|c|c|c|c|}
\hline & 2008 & 2009 & 2010 & 2011 & 2012 & 2013 & 2014 & 2015 & 2016 & 2017 \\
\hline 2008 & 1.00 & 0.91 & 0.86 & 0.84 & 0.84 & 0.80 & 0.81 & 0.80 & 0.80 & 0.78 \\
\hline 2009 & 0.91 & 1.00 & 0.91 & 0.88 & 0.87 & 0.85 & 0.86 & 0.85 & 0.83 & 0.82 \\
\hline 2010 & 0.86 & 0.91 & 1.00 & 0.90 & 0.88 & 0.89 & 0.89 & 0.87 & 0.85 & 0.85 \\
\hline 2011 & 0.84 & 0.88 & 0.90 & 1.00 & 0.94 & 0.91 & 0.92 & 0.91 & 0.89 & 0.89 \\
\hline 2012 & 0.84 & 0.87 & 0.88 & 0.94 & 1.00 & 0.94 & 0.94 & 0.92 & 0.90 & 0.90 \\
\hline 2013 & 0.80 & 0.85 & 0.89 & 0.91 & 0.94 & 1.00 & 0.95 & 0.92 & 0.91 & 0.91 \\
\hline 2014 & 0.81 & 0.86 & 0.89 & 0.92 & 0.94 & 0.95 & 1.00 & 0.94 & 0.93 & 0.93 \\
\hline 2015 & 0.80 & 0.85 & 0.87 & 0.91 & 0.92 & 0.92 & 0.94 & 1.00 & 0.93 & 0.93 \\
\hline 2016 & 0.80 & 0.83 & 0.85 & 0.89 & 0.90 & 0.91 & 0.93 & 0.93 & 1.00 & 0.95 \\
\hline 2017 & 0.78 & 0.82 & 0.85 & 0.89 & 0.90 & 0.91 & 0.93 & 0.93 & 0.95 & 1.00 \\
\hline
\end{tabular}

Table 4. Results from the application ICA by years over the input data - degree of membership 


\begin{tabular}{|c|c|c|c|c|c|c|c|c|c|c|}
\hline & 2008 & 2009 & 2010 & 2011 & 2012 & 2013 & 2014 & 2015 & 2016 & 2017 \\
\hline 2008 & 0.00 & 0.06 & 0.12 & 0.14 & 0.13 & 0.17 & 0.17 & 0.17 & 0.18 & 0.20 \\
\hline 2009 & 0.06 & 0.00 & 0.06 & 0.10 & 0.10 & 0.13 & 0.12 & 0.12 & 0.14 & 0.15 \\
\hline 2010 & 0.12 & 0.06 & 0.00 & 0.09 & 0.09 & 0.10 & 0.10 & 0.11 & 0.13 & 0.13 \\
\hline 2011 & 0.14 & 0.10 & 0.09 & 0.00 & 0.04 & 0.07 & 0.07 & 0.07 & 0.10 & 0.10 \\
\hline 2012 & 0.13 & 0.10 & 0.09 & 0.04 & 0.00 & 0.04 & 0.05 & 0.06 & 0.08 & 0.09 \\
\hline 2013 & 0.17 & 0.13 & 0.10 & 0.07 & 0.04 & 0.00 & 0.04 & 0.06 & 0.08 & 0.08 \\
\hline 2014 & 0.17 & 0.12 & 0.10 & 0.07 & 0.05 & 0.04 & 0.00 & 0.04 & 0.06 & 0.06 \\
\hline 2015 & 0.17 & 0.12 & 0.11 & 0.07 & 0.06 & 0.06 & 0.04 & 0.00 & 0.05 & 0.05 \\
\hline 2016 & 0.18 & 0.14 & 0.13 & 0.10 & 0.08 & 0.08 & 0.06 & 0.05 & 0.00 & 0.04 \\
\hline 2017 & 0.20 & 0.15 & 0.13 & 0.10 & 0.09 & 0.08 & 0.06 & 0.05 & 0.04 & 0.00 \\
\hline
\end{tabular}

Table 5. Results from the application ICA by years over the input data degree of non-membership

The results of the ICA application for determining the correlations of the data for Human Resources in Science and Technology in years are investigated and the following outcomes are obtained: 2 pairs of years in strong positive consonance, 34 pairs of years in positive consonance and 9 pairs of years in weak positive consonance. The intuitionistic fuzzy triangle interpretation with the results of the ICA application is presented on Fig. 2.

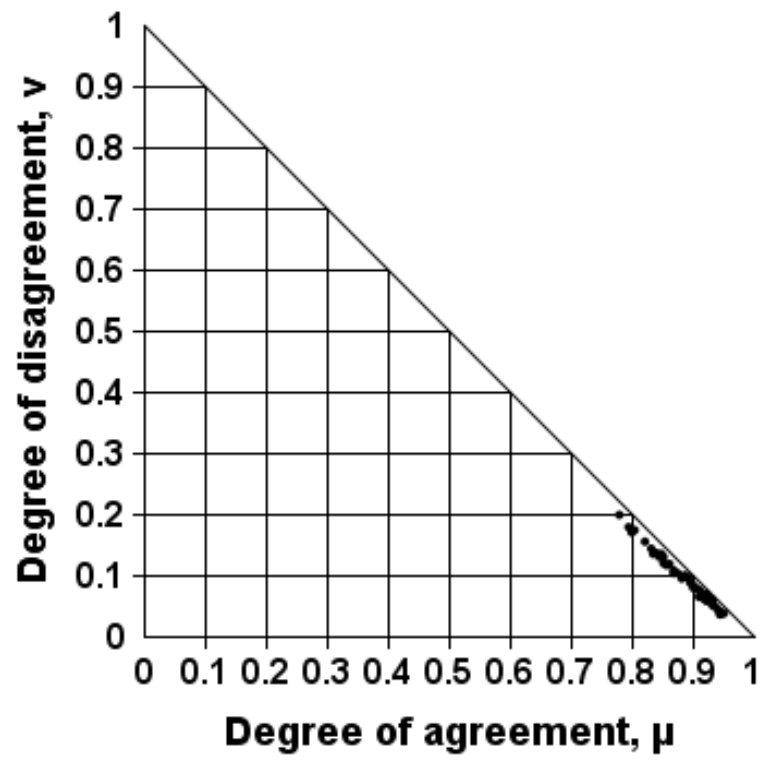

Figure 2. Results from application of ICA for determining the correlations between the years

The pairs having the most similar trend of Human Resources in Science \& Technology are the years 2013-2014 and 2016-2017. The ICA application for determining the correlation between the years presents the similar and accurate tendency of Human Resources in Science $\&$ Technology. 


\section{Conclusion}

In the presented paper we used the ICA approach to analyze data from HRST as part from Eurostat statistical information. After a successful application and following the results, we can conclude that intuitionistic fuzzy pairs in dissonance have the highest independence level. The pairs in weak dissonance are independent with slight hesitations in-between. The criteria in weak positive consonance and mostly positive consonance, they can be considered close, but not interchangeable. The pairs of criteria in positive consonance and strong positive consonance have expressed dependencies.

\section{Acknowledgment}

The authors are grateful for the support provided by the project "Intelligent Instruments for Knowledge Discovering and Processing" (No. NIH-418/2018).

\section{References}

[1] Angelova, M., Roeva, O., \& Pencheva, T. (2015). InterCriteria Analysis of a cultivation process model based on the genetic algorithm population size influence, Notes on Intuitionistic Fuzzy Sets, 21 (4), 90-103.

[2] Atanassov K., Ribagin, S., Sotirova, E., Bureva, V., Atanassova, V., \& Angelova, N. (2017). Intercriteria analysis using special type of intuitionistic fuzzy implications, Notes on Intuitionistic Fuzzy Sets, 23 (5), 61-65.

[3] Atanassov, K. (2012). On Intuitionistic Fuzzy Sets Theory, Springer, Berlin.

[4] Atanassov, K. (2014). Index Matrices: Towards an Augmented Matrix Calculus. Studies in Computational Intelligence Series, Vol. 573, Springer, Cham.

[5] Atanassov, K., Atanassova, V., \& Gluhchev, G. (2015). InterCriteria Analysis: ideas and problems, Notes on Intuitionistic Fuzzy Sets, 21 (1), 81-88.

[6] Atanassov, K., Mavrov, D., \& Atanassova, V. (2014). InterCriteria Decision making: a new approach for multicriteria decision making, based on index matrices and intuitionistic fuzzy sets. Issues in Intuitionistic Fuzzy Sets and Generalized Nets, 11, 1-8.

[7] Atanassov, K., Szmidt, E., \& Kacprzyk, J. (2013). On intuitionistic fuzzy pairs, Notes on Intuitionistic Fuzzy Sets, 19 (3), 1-13.

[8] Atanassova, V., Doukovska, L., Atanassov, K., \& Mavrov, D. (2014). InterCriteria decision making approach to eu member states competitiveness analysis, Proc. of the Int.Symp. on Business Modeling and Software Design - BMSD’14, 289-294.

[9] Atanassova, V., Doukovska, L., Karastoyanov, D., \& Capkovic, F. (2014). InterCriteria decision making approach to eu member states competitiveness analysis: trend analysis. 
Proceedings of IEEE Intelligent Systems'2014, Advances in Intelligent Systemsand Computing, Vol. 322, Springer, 107-115.

[10] Atanassova, V., Doukovska, L., Mavrov, D., \& Atanassov, K. (2014). InterCriteria decision making approach to eu member states competitiveness analysis: temporal and threshold analysis. Proceedings of IEEE Intelligent Systems'2014, Advances inIntelligent Systems and Computing, Vol. 322, Springer, 95-106.

[11] Atanassova, V., Doukovska, L., Michalikova, A., \& Radeva, I. (2016), I. Intercriteria analysis: From pairs to triples. Notes on Intuitionistic Fuzzy Sets, 22 (5), 98-110.

[12] Bureva, V., Michalíková A., Sotirova E., Popov S., Riečan B., Roeva O. (2017). Application of the InterCriteria Analysis to the universities rankings system in the Slovak Republic, 21st ICIFS, 22-23 May 2017, Burgas, Bulgaria, Notes on Intuitionistic Fuzzy Sets, 23 (2), 128-140.

[13] Bureva, V., Sotirova E., Atanassova V., Angelova N., \& Atanassov K. (2018) Intercriteria Analysis over Intuitionistic Fuzzy Data. In: Lirkov I., Margenov S. (eds) Large-Scale Scientific Computing. LSSC 2017. Lecture Notes in Computer Science, Vol. 10665. Springer, Cham, .333-340

[14] Bureva, V., Sotirova, E., Sotirov, S., \& Mavrov, D. (2015). Application of the InterCriteria decision making method to Bulgarian universities ranking, Notes onIntuitionistic Fuzzy Sets, 21 (2), 111-117.

[15] Eurostat: Education and Training Database, Human Resources in Science \& Technology (2019, 23 April). Retrieved from: https://ec.europa.eu/eurostat/web/ science-technology-innovation/data/database.

[16] Ikonomov, N., Vassilev P., \& Roeva O. (2018). ICrAData - Software for InterCriteria Analysis, Int.J.Bioautomation, 22 (1), 1-10.

[17] Petkova, S., Tasheva, Y., Petkov, P. (2009). Trends in Development of the Petrol Refining Industry in the World. I part, The Questions about the Ratio between World Yield of Oil and the Capacities of Its Refining, Oxidation Communications, 32 (1), 146-156.

[18] Petkova, S., Tasheva, Y., Petkov, P. (2010). Classification and possibilities of management of primary energy sources, Oxidation Communications, 33 (2), 462-469.

[19] Sotirov, S., Atanassova, V., Sotirova, E., Doukovska, L., Bureva, V., Mavrov, D., \& Tomov, J. (2017). Application of the Intuitionistic Fuzzy InterCriteria Analysis Method with Triples to a Neural Network Preprocessing Procedure, Computational Intelligence and Neuroscience Volume 2017, Article ID 2157852, 9 pages, doi:10.1155/2017/21578524.

[20] Stratiev, D., Sotirov, S., Shishkova, I., Nedelchev, A., Sharafutdinov, I., Veli, A., Mitkova, M., Yordanov, D., Sotirova, E., Atanassova, V., Atanassov, K., Stratiev, D., Rudnev, N., \& Ribagin, S. (2016). Investigation of relationships between bulk properties and fraction properties of crude oils by application of the InterCriteria Analysis, Petroleum Science and Technology, 34 (13), 1113-1120. 
[21] Traneva, V., Tranev, S., Szmidt, E., \& Atanassov, K. (2018) Three Dimensional Intercriteria Analysis over Intuitionistic Fuzzy Data. Advances in Intelligent Systems and Computing, 643, 442-449.

[22] Vankova, D., Sotirova, E., \& Bureva, V. (2015). An application of the InterCriteria Analysis approach to health-related quality of life, Notes on Intuitionistic Fuzzy Sets, 21 (5), 40-48.

[23] Zadeh, L. A. (1965) Fuzzy Sets. Information and Control, 8, 333-353. 